\title{
Amostragem para avaliação da fertilidade do solo em função da variabilidade de suas características químicas
}

\author{
Hemmannuella C. Santos ${ }^{1}$, Fábio H. T. de O liveira ${ }^{2}$, Jandeilson A. de Arruda ${ }^{3}$, \\ Almir R. da S. Lopes ${ }^{4}$, Reginaldo F. de Souza Júnior ${ }^{5} \&$ Daise R. de Farias ${ }^{5}$
}

\begin{abstract}
RESUMO
Visou-se, neste trabal ho, avaliar a variabilidade de características químicas do solo, definir o número de amostras simples para formar uma amostra composta e avaliar se a média aritmética das amostras simples é igual ao resultado da análise química da amostra composta. Realizaram-se três séries de amostragens constituídas de 36 amostras simples por série, em um Latossolo Amarelo. 0 s locais de retirada das amostras da primeira série se distanciaram $15 \mathrm{~m}$ um do outro, os da segunda série, 2,5 m, e os da terceira série, 0,42 $\mathrm{m}$. Com as amostras de solo da primeira série, foram formuladas amostras compostas mediante a mistura homogênea de 4, 8, 14, 20, 28 e 36 amostras simples, utilizando-se três repetições. A variabilidade das características químicas do solo praticamente não variou quando o tamanho da área amostrada variou de $8.100 \mathrm{~m}^{2}$ para $225 \mathrm{~m}^{2}$. Para recomendação de calagem e adubação, as doses de calcário e fertilizante são, em geral, as mesmas, independentemente se a fertilidade média do solo é avaliada a partir da análise química da amostra composta ou a partir da média aritmética dos resultados das análises químicas das amostras simples. A coleta de pelo menos oito amostras simples de solo foi suficiente para formar uma amostra composta representativa.
\end{abstract}

Palavras-chave: amostra composta, amostra simples, análise de solo

\section{Soil sampling for evaluation of fertility as a function of variability of chemical characteristics}

\begin{abstract}
The aim of this study was to evaluate the variability of chemical attributes of soil, to define the number of single samples required to form a composite sample and to evaluate if the mean fertility values obtained from the composite sample is the same as obtained from the arithmetic mean of single samples. Three series of sampling, each one with 36 samples collected in an 0 xisol, were carried out. Samples of the first series were taken $15 \mathrm{~m}$ apart from each other, the second one $2.5 \mathrm{~m}$, and the third one $0.42 \mathrm{~m}$. Composite samples were formulated with samples of first series by a homogenous mixture of 4, 8, 14, 20,28 e 36 single samples, with three replications. The variability of soil chemical attributes did not vary when the area size decreased from $8,100 \mathrm{~m}^{2}$ to $225 \mathrm{~m}^{2}$. D oses of lime and fertilizer may have the same recommendation, independently if soil fertility was evaluated by chemical analysis of a composite sample or obtained from the arithmetic mean of single sampling. At least eight single samples were necessary to compose a representative sample of the area.
\end{abstract}

Key words: composite sample, single sample, soil analysis

\footnotetext{
${ }^{1}$ D outoranda do Programa de Pós-Graduação em Agronomia da UFPB. CEP 58397-000. Areia, PB. Fone (83) 9139-0258, E-mail: hecosantos@yahoo.com.br 2 DCAT/U FERSA. CEP 59.625-190. Mossoró, RN. Fone: (84) 3315-1741. E-mail: fabio@ufersa.edu.br

${ }^{3}$ Mestrando em Solos e Nutrição de Plantas/UFV. CEP 36570-000. Viçosa, MG. Fone (31) 8686-6054 E-mail: jandeilson.arruda@ufv.br

${ }^{4} \mathrm{G}$ raduando do CCA/U FPB. CEP 58397-000. Areia, PB. Fone (83) 3362-2300, E-mail: kaliroger@bol.com.br

${ }_{5}^{5}$ Mestrando (a) em Manejo de Solo e Água do CCA/U FPB. CEP 58397-000. Areia, PB. Fone (83) 8825-9857, E-mail: agroregi@yahoo.com.br
} 


\section{INTRODUÇãO}

Considera-se que as características químicas do solo variam quanto à distância em três escalas (James \& Wells, 1990): (1) macrovariações - quando a distância entre dois pontos de amostragem é superior a 2,00 m; (2) mesovariações - indicam variações entre pontos de amostragem separados de 0,05 a 2,00 m e (3) microvariações - referem-se às variações entre pontos de amostragem com distância menor que $5 \mathrm{~cm}$.

As macrovariações são resultantes da ação dos processos pedogenéticos que atuaram sobre diferentes materiais de origem, gerando solos diferentes quanto à cor, topografia, textura, vegetação, morfologia, mineralogia, qualidade e quantidade de matéria orgânica e disponibilidade de água e de nutrientes. Essas macrovariações normalmente se correlacionam espacialmente, apresentando valores elevados de alcance da dependência espacial (Mello et al., 2006). As meso e microvariações ocorrem naturalmente, por acréscimos localizados e aleatórios de restos vegetais e animais, porém podem ser intensificadas em decorrência da adoção de práticas de manejo, como a distância entre as linhas de cultivo e as doses de fertilizantes. Neste caso, as características químicas do solo tendem a apresentar distribuição espacial aleatória e normalmente elas são intensas e variam a curta distância (Oliveira et al., 2002).

As recomendações de calagem e adubação para as culturas são feitas com base na interpretação de análises químicas de amostras compostas de solo, partindo-se do princípio de que o resultado de uma amostra composta representa a fertilidade média da área a ser amostrada. A representatividade adequada da amostra composta está diretamente relacionada com a qualidade das amostras simples (Guarçoni M. et al., 2006, 2007). Entre outros requisitos, exige-se que as amostras simples sejam coletadas na mesma profundidade, possuam mesmo volume e reflitam as condições de fertilidade de seus respectivos sítios de coleta; além disso, quanto maior a variabilidade das características químicas do solo maior também deverá ser o número de amostras simples para formar uma amostra composta representativa.

O uso da amostra composta de solo para avaliação da fertilidade do solo se baseia na hipótese de que a fertilidade média estimada a partir da análise química da amostra composta é semelhante àquela estimada a partir da média aritmética dos resultados das amostras simples (Alvarez V. \& Guarçoni M., 2003; Oliveira et al., 2007).

O número de amostras simples para formar uma amostra composta representativa depende sobretudo do erro admitido em torno da média verdadeira e da variabilidade dos índices de fertilidade do solo (Alvarez V. \& Carraro, 1976; Schlindwein \& Anghinoni, 2000; Oliveira et al., 2002; Alvarez V. \& Guarçoni M., 2003), levando-se em conta que a inexistência de dependência espacial possibilita o uso da estatística clássica para descrever o fenômeno. Ao assumir independência entre as amostras dentro da unidade de amostragem, garante-se o uso da média e do desvio padrão para representar o fenômeno, já que valores próximos não são necessariamente mais parecidos entre si do que valores distantes.

A variabilidade de características químicas do solo dentro de parcelas de diferentes tamanhos em unidades de amostragem aparentemente homogêneas de dois Latossolos (LVm e LR) foi estudada no Estado do Paraná (Alvarez V. \& Carraro, 1976); em cada uma das duas unidades se realizaram três séries de amostragem, constituídas de 49 amostras simples por série; as amostras da primeira série guardaram a distância de 14,7 m uma da outra, as da segunda série, 2,1 m e as da terceira série, $0,3 \mathrm{~m}$. Os dados indicaram que, para $\mathrm{pH}$ e teores de $\mathrm{P}, \mathrm{K}^{+}, \mathrm{Al}^{3+}, \mathrm{Ca}^{2+}, \mathrm{Mg}^{2+}$ e matéria orgânica, o LVm de Ponta Grossa foi mais uniforme do que o LR de Cascavel; em geral, no LR a variabilidade dessas características diminuiu a medida em que se diminuiu a distância entre pontos amostrados, sendo mais marcante quando se passou de 14,7 para 2,1 $\mathrm{m}$ do que quando se passou de 2,1 para $0,3 \mathrm{~m}$; no $\mathrm{LVm}$, para $\mathrm{pH}, \mathrm{Pe} \mathrm{K}^{+}$, a variabilidade na série a $14,7 \mathrm{~m}$ foi semelhante à da série a $0,3 \mathrm{~m}$ e menor que na série a $2,1 \mathrm{~m}$.

Caso se admita que a variabilidade diminui com a diminuição do tamanho da área, o número de amostras simples para formar uma amostra composta representativa de uma parcela (área pequena) de um experimento de campo pode ser menor que o número de amostras simples para formar uma amostra composta representativa da área experimental (área grande) que contém esta parcela. Guarçoni M. et al. (2006), porém, observaram que a variabilidade dos índices de fertilidade do solo em uma área de $1,288 \mathrm{~m}^{2}$ foi semelhante à variabilidade estimada para uma grande área de solo (talhão aparentemente homogêneo), demonstrando que a variabilidade é intensa e ocorre a curtas distâncias no solo; espera-se, portanto, que o número de amostras simples necessário para formar uma amostra composta representativa seja o mesmo não apenas em uma parcela experimental mas também em um talhão aparentemente homogêneo.

Propôs-se, com este trabalho, medir a variabilidade das principais características químicas do solo e testar as hipóteses de que a mesma não diminui com a diminuição do tamanho da unidade de amostragem (talhão aparentemente homogêneo) e, ainda, que a fertilidade média estimada a partir da média aritmética dos resultados das amostras simples é semelhante àquela estimada a partir da análise química da amostra composta.

\section{MATERIAL E MÉTODOS}

Amostras de solo foram coletadas em uma unidade de amostragem aparentemente homogênea, de aproximadamente um hectare, em um Latossolo Amarelo, localizado no município de Areia, $\mathrm{PB}$, sob pastagem e sem adubação.

Dentro dessa unidade de amostragem foi demarcada uma área de 90 x $90 \mathrm{~m}$, que foi dividida em 36 quadrados de $15 \mathrm{~m}$ de lado; no centro de cada quadrado se retirou uma amostra simples de uns $1500 \mathrm{~g}$ de solo na camada de $0-20 \mathrm{~cm}$ de profundidade, com o auxílio de um enxadeco, perfazendo o total 
de 36 amostras simples. Em um quadrado (15 x 15 m) escolhido ao acaso entre os 36 já descritos, realizou-se nova divisão em 36 quadrados de $2,5 \mathrm{~m}$ de lado e, no centro de cada um desses quadrados, se coletou uma amostra simples de solo de aproximadamente $1500 \mathrm{~g}$, na profundidade de 0-20 cm, perfazendo o total de 36 amostras simples. Em um quadrado de 2,5 x 2,5 m, limitado pelos pontos de amostragem de quatro amostras próximas e previamente selecionado ao acaso, realizou-se nova divisão em 36 quadrados de 0,42 m de lado, retirando-se a mesma quantidade de solo; ao todo, foram coletadas 108 amostras simples de solo, sendo que os locais de coleta das 36 amostras de solo da primeira série de amostragem guardaram a distância de $15 \mathrm{~m}$ um do outro, os das 36 amostras da segunda série guardaram um do outro a distância de $2,5 \mathrm{~m}$ e os locais de coleta das 36 amostras de solo da terceira série de amostragem foram distanciados $0,42 \mathrm{~m}$ um do outro (Figura 1).

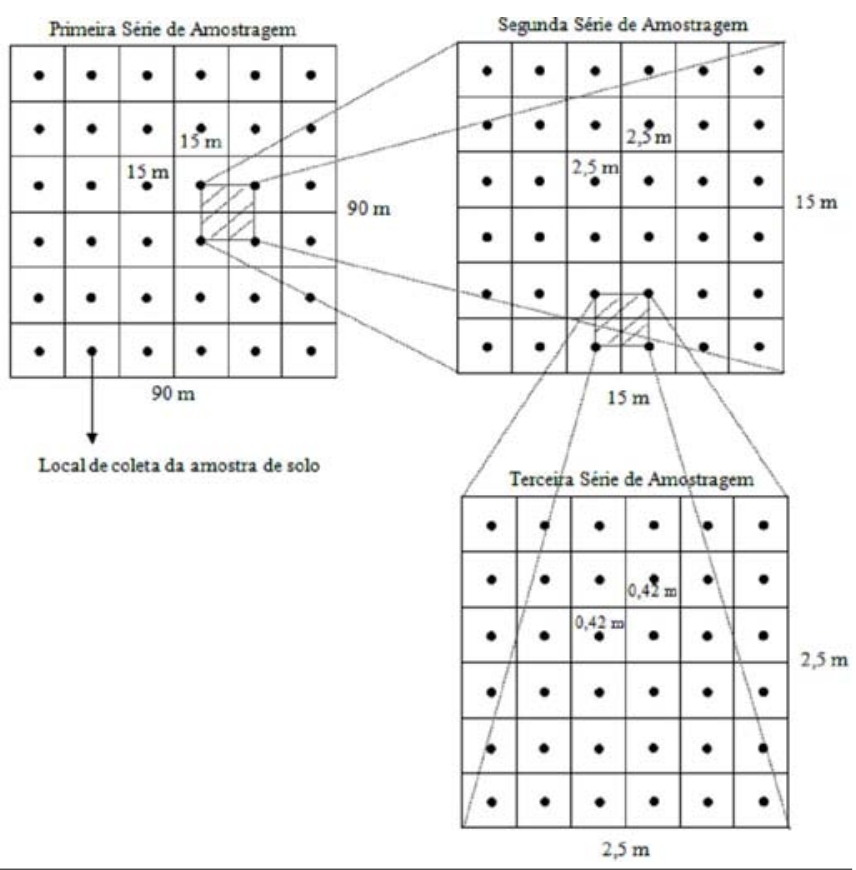

Figura 1. Croqui para identificação dos locais de coleta de amostras simples de solo

As amostras de solo foram secadas ao ar e passadas em peneira de $2 \mathrm{~mm}$ de malha e encaminhadas para o Laboratório de Química e Fertilidade do Solo do Departamento de Solos e Engenharia Rural do Centro de Ciências Agrárias da Universidade Federal da Paraíba, para determinação do $\mathrm{pH}$ e dos teores de matéria orgânica (M.O), $\mathrm{P}^{+} \mathrm{K}^{+}, \mathrm{Ca}^{2+}$, $\mathrm{Mg}^{2+} \mathrm{e} \mathrm{Al}^{3+}$.

Foram calculados, para cada uma das três séries de amostragem, a média, a variância e o coeficiente de variação de todas as características químicas avaliadas; em seguida, foram testadas as diferenças entre as variâncias e entre as médias de todas as características químicas, pelos testes $\mathrm{F}$ de Fisher e $\mathrm{t}$ de Student, respectivamente. Determinouse, também, o número mínimo de amostras simples necessário para formar uma amostra composta representativa, através da fórmula (Barreto et al., 1974; Cantarutti et al.,
2007): $\mathrm{n}=\left[\left(\mathrm{t}_{\mathrm{tab}} \cdot \mathrm{CV}\right) / \mathrm{f}\right]^{2}$, donde $\mathrm{n}$ é o número ideal de amostras simples necessária para a formação de uma amostra composta representativa, $\mathrm{t}_{\mathrm{tab}}$ é o valor de t tabelado para $\alpha=5 \%$ (bilateral) e G.L $=35, \mathrm{CV}$ é o coeficiente de variação da característica avaliada e f é o erro admitido em torno da média verdadeira $(20 \%)$.

A partir da Terra Fina Seca ao Ar (TFSA) das 36 amostras simples de solo da primeira série de amostragem foram retiradas, ao acaso, sub-amostras para simular a formação de seis tipos de amostras compostas formadas por diferentes números de amostras simples, sendo: 3 compostas de 4 simples, 3 de 8,3 de 14, 3 de 20, 3 de 28 e 3 compostas de 36 simples, para determinação do $\mathrm{pH}$ e dos teores de M.O, $\mathrm{P}, \mathrm{K}^{+}, \mathrm{Ca}^{2+}, \mathrm{Mg}^{2+}, \mathrm{Al}^{3+} \mathrm{e}(\mathrm{H}+\mathrm{Al})$. Para testar a hipótese de que os valores médios desses índices de fertilidade do solo calculados a partir da média aritmética dos resultados das amostras simples são semelhantes àqueles estimados a partir da análise química da amostra composta, realizou-se um experimento no delineamento inteiramente casualizado, contendo 12 tratamentos e 3 repetições, totalizando 36 unidades experimentais. Os tratamentos resultaram da combinação fatorial entre seis "tamanhos de amostras compostas" $(4,8,14,20,28$ e 36 amostras simples para formar uma amostra composta) x duas formas de avaliação do valor médio dos índices de fertilidade do solo (média aritmética dos atributos analisados nas amostras simples e análise química da amostra composta). Testaram-se, por meio de análise de variância e de regressão, os efeitos dos fatores em estudo.

\section{RESULTADOS E DISCUSSÃO}

Quando o tamanho da área amostrada diminuiu de 8.100 para $225 \mathrm{~m}^{2}$, os teores médios de $\mathrm{Ca}^{2+}$ e de M.O diminuíram mas não houve variação da média dos teores de $\mathrm{P}, \mathrm{K}^{+}, \mathrm{Mg}^{2+}$, $\mathrm{Al}^{3+}$ e da média dos valores de $\mathrm{pH}$ (Tabela 1). Quando o tamanho da área amostrada passou de 225 para $6,25 \mathrm{~m}^{2}$, as médias para os teores de $\mathrm{Ca}^{2+}, \mathrm{Mg}^{2+}$, M.O e para o $\mathrm{pH}$ permaneceram constantes, enquanto as médias dos teores de $\mathrm{K}^{+}$e de $\mathrm{Al}^{3+}$ diminuíram e a média do teor de $\mathrm{P}$ aumentou (Tabela 1).

Para $\mathrm{P}, \mathrm{Mg}^{2+}, \mathrm{Al}^{3+}$ e $\mathrm{pH}$, as variâncias não mudaram em nenhuma situação de variação da distância entre os pontos amostrados (Tabela 1); quando a distância entre pontos amostrados diminuiu de 15 para $2,5 \mathrm{~m}$, as variâncias dos teores de $\mathrm{Ca}^{2+}$ e de M.O diminuíram mas a dos teores de $\mathrm{K}^{+}$se manteve constante (Tabela 1); quando o tamanho da área amostrada diminuiu de 225 para $6,25 \mathrm{~m}^{2}$, as variâncias das características químicas não mudaram, com exceção da variância do teor de $\mathrm{K}^{+}$que diminuiu (Tabela 1 ).

O padrão de variação da média e da variância das características químicas do solo em função do tamanho da área amostrada observado neste trabalho, indica que o número de amostras simples para formar uma amostra composta representativa de uma pequena parcela de um experimento de campo (área $\cong 225 \mathrm{~m}^{2}$ ) deve ser igual ao número de amostras simples para formar uma amostra composta representativa de 
Tabela 1. Média, variância e coeficiente de variação para os teores de fósforo, potássio, cálcio, magnésio, alumínio, matéria orgânica e para 0 pH, em função do tamanho da unidade de amostragem

\begin{tabular}{|c|c|c|c|}
\hline \multirow{2}{*}{ Parâmetro } & \multicolumn{3}{|c|}{$\begin{array}{l}\text { Tamanho da unidade de amostragem }\left(\mathrm{m}^{2}\right) / \\
\text { distância entre os pontos amostrados }(\mathrm{m})\end{array}$} \\
\hline & $8.100 / 15$ & $225 / 2,5$ & $6,25 / 0,42$ \\
\hline \multicolumn{4}{|c|}{ Teor de fósforo disponível $\left(\mathrm{mg} \mathrm{dm}^{-3}\right)$} \\
\hline Média ${ }^{(1)}$ & $2,27 \mathrm{~b}$ & $2,25 \mathrm{~b}$ & $2,40 a$ \\
\hline Variância ${ }^{(2)}$ & 0,097 a & $0,197 \mathrm{a}$ & $0,114 \mathrm{a}$ \\
\hline Coeficiente de variação & 13,7 & 19,7 & 14,0 \\
\hline $\begin{array}{l}\text { "Tamanho ideal" da amostra } \\
\text { composta }^{(3)}\end{array}$ & 2 & 4 & 2 \\
\hline \multicolumn{4}{|c|}{ Teor de potássio disponível $\left(\mathrm{mg} \mathrm{dm}^{-3}\right)$} \\
\hline Média(1) & $29,42 a$ & 25,92 a & $20,96 b$ \\
\hline Variância ${ }^{(2)}$ & $211,058 \mathrm{a}$ & $232,639 a$ & $51,443 b$ \\
\hline Coeficiente de variação & 49,4 & 58,9 & 34,2 \\
\hline $\begin{array}{l}\text { "Tamanho ideal" da amostra } \\
\text { composta }^{(3)}\end{array}$ & 25 & 36 & 12 \\
\hline \multicolumn{4}{|c|}{ Teor de cálcio trocável $\left(\mathrm{cmol}_{\mathrm{c}} \mathrm{dm}^{-3}\right)$} \\
\hline Média(1) & $0,76 \mathrm{a}$ & $0,39 \mathrm{~b}$ & $0,40 \mathrm{~b}$ \\
\hline $\operatorname{Variância(2)~}^{(2)}$ & 0,257 a & $0,047 \mathrm{~b}$ & $0,019 b$ \\
\hline Coeficiente de variação & 67,11 & 56,12 & 34,07 \\
\hline $\begin{array}{l}\text { "Tamanho ideal" da amostra } \\
\text { composta }^{(3)}\end{array}$ & 46 & 32 & 12 \\
\hline \multicolumn{4}{|c|}{ Teor de magnésio trocável $\left(\mathrm{cmol}_{\mathrm{c}} \mathrm{dm}^{-3}\right)$} \\
\hline Média(1) & $0,46 \mathrm{a}$ & $0,40 \mathrm{~b}$ & $0,44 a b$ \\
\hline Variância(2) & $0,028 \mathrm{a}$ & $0,020 \mathrm{a}$ & $0,019 a$ \\
\hline Coeficiente de variação & 35,76 & 35,34 & 31,34 \\
\hline $\begin{array}{l}\text { "Tamanho ideal" da amostra } \\
\text { composta }^{(3)}\end{array}$ & 13 & 13 & 10 \\
\hline \multicolumn{4}{|c|}{ Teor de alumínio trocável $\left(\mathrm{cmol}_{c} \mathrm{dm}^{-3}\right)$} \\
\hline Média(1) & $1,13 \mathrm{~b}$ & $1,45 a$ & $1,22 \mathrm{~b}$ \\
\hline Variância(2) & $0,099 \mathrm{a}$ & 0,074 a & $0,039 a$ \\
\hline Coeficiente de variação & 27,70 & 18,72 & 16,14 \\
\hline $\begin{array}{l}\text { "Tamanho ideal" da amostra } \\
\text { composta }^{(3)} \\
\end{array}$ & 8 & 4 & 3 \\
\hline \multicolumn{4}{|c|}{ Teor de matéria orgânica $\left(\mathrm{g} \mathrm{kg}^{-1}\right)$} \\
\hline Média(1) & $27,23 \mathrm{~b}$ & 31,08 a & $31,27 \mathrm{a}$ \\
\hline $\operatorname{Variância(2)~}^{(2)}$ & $33,036 \mathrm{a}$ & $5,253 \mathrm{~b}$ & $4,130 \mathrm{~b}$ \\
\hline Coeficiente de variação & 21,10 & 7,37 & 6,50 \\
\hline $\begin{array}{l}\text { "Tamanho ideal" da amostra } \\
\text { composta }^{(3)}\end{array}$ & 5 & 1 & 1 \\
\hline \multicolumn{4}{|l|}{ (t) } \\
\hline Média(1) & $4,80 \mathrm{a}$ & 4,76 a & 4,74 a \\
\hline Variância $^{(2)}$ & $0,048 \mathrm{a}$ & $0,033 a$ & $0,049 a$ \\
\hline Coeficiente de variação & 4,55 & 3,81 & 4,66 \\
\hline $\begin{array}{l}\text { "Tamanho ideal" da amostra } \\
\text { composta(3) }\end{array}$ & 1 & 1 & 1 \\
\hline
\end{tabular}

${ }^{(1)}$ Médias seguidas de mesma letra na linha não diferem entre si pelo teste $\mathrm{t}$ de Student a $5 \%$

(2)Variâncias seguidas de mesma letra na linha não diferem entre si pelo teste $\mathrm{F}$

${ }^{(3)}$ Refere-se ao número ideal de amostras simples a serem retiradas para formar uma amostra composta representativa, calculado pela expressão $\mathrm{n}=\left[\left(\mathrm{t}_{\mathrm{tab}} \cdot \mathrm{CV}\right) / \mathrm{f}\right]^{2}$, em que $\mathrm{t}_{\text {tab }}$ é o valor de $\mathrm{t}$ tabelado para $\mathrm{a}=5 \%$ e G.L $=35, \mathrm{CV}$ é o coeficiente de variação da característica avaliada e f é o erro admitido em torno da média verdadeira (20\%)

uma grande unidade de amostragem aparentemente homogênea (área $\cong 8.100 \mathrm{~m}^{2}$ ). Para Raij (1991), o número de amostras simples para formar uma amostra composta deve ser o mesmo, qualquer que seja a área a ser amostrada, mesmo que ela represente apenas $10 \mathrm{~m}^{2}$. Segundo Cantarutti et al. (2007), para uma grande área (centenas de hectares) ou para uma pequena área de uma parcela experimental, por exemplo, o número de amostras para caracterizar a fertilidade média do solo seria o mesmo. $\mathrm{O}$ fato das variâncias não terem mudado quando o tamanho da área diminuiu de 225 para $6,25 \mathrm{~m}^{2}$, também indica que a variabilidade das características químicas do solo ocorre a curtas distâncias, o que está de acordo com Alvarez V. \& Carraro (1976), Saraiva et al. (1992), Raun et al. (1998) e Guarçoni M. et al. (2006; 2007).

O coeficiente de variação tem sido a medida mais utilizada para quantificar a variabilidade das características químicas do solo (Schlindwein \& Anghinoni, 2000; Alvarez V. \& Guarçoni M., 2003; Guarçoni M. et al., 2006; 2007; Silva et al., 2007). De modo geral, esses trabalhos mostram que o P e o $\mathrm{K}^{+}$são as características químicas que apresentam maiores valores de coeficientes de variação e a M.O e o $\mathrm{pH}$, os menores valores; entretanto, neste trabalho se verificou a seguinte sequência decrescente de variabilidade dos índices de fertilidade do solo: $\mathrm{Ca}^{2+}>\mathrm{K}^{+}>\mathrm{Mg}^{2+}>\mathrm{Al}^{3+}$ $>\mathrm{M} . \mathrm{O}>\mathrm{P}>\mathrm{pH}$. Também carecem de atenção, neste trabalho, os valores de coeficientes de variação muito baixos para os teores de $\mathrm{P}$ no solo, quando o esperado é que este índice de fertilidade do solo apresente a maior variabilidade (Machado et al., 2007).

A menor variabilidade do $\mathrm{P}$ em relação às outras variáveis apresentadas neste trabalho, se deve ao fato de que, das 108 amostras analisadas, 56\% apresentaram valor igual a $2,08 \mathrm{mg} \mathrm{dm}^{-3}, 22 \%$ valor igual a $2,45 \mathrm{mg} \mathrm{dm}^{-3}, 14 \%$ valor igual $2,76 \mathrm{mg} \mathrm{dm}^{-3}$ e os $8 \%$ restantes outros valores, todos muito próximos ao limite de detecção do método analítico empregado pelo laboratório para determinação do teor de $P$ disponível no solo. Esses valores muito baixos se devem ao fato do solo onde foram coletadas as amostras ser naturalmente muito pobre em $\mathrm{P}$ e não ter recebido aplicação de adubo fosfatado.

Em geral, as médias dos teores de $\mathrm{K}^{+}$e dos valores de $\mathrm{pH}$ do solo foram as mesmas, independentemente se elas foram avaliadas a partir da média aritmética dos valores obtidos nas amostras simples ou se foram estimadas a partir da análise química da amostra composta (Tabelas 2 e 3); no entanto, para os teores de $\mathrm{P}, \mathrm{Ca}^{2+}, \mathrm{Mg}^{2+}, \mathrm{Al}^{3+}$ e M.O., os valores médios encontrados nos dois métodos citados diferiram entre si e, para $\mathrm{Ca}^{2+} \mathrm{e} \mathrm{Al}^{3+}$, o método da média aritmética superou o método da amostra composta em cerca de 15 e $10 \%$, respectivamente. Para $\mathrm{Mg}^{2+}$ e M.O, os valores encontrados na amostra composta superaram $(40 \%$ para $\mathrm{Mg}^{2+}$ e $16 \%$ para M.O) aqueles obtidos a partir da média aritmética dos resultados encontrados nas amostras simples (Tabelas 2 e 3). A diferença encontrada entre as duas formas de obtenção do teor médio de $\mathrm{P}$ disponível (Tabela 3) é de pequena magnitude $\left(0,1 \mathrm{mg} \mathrm{dm}^{-3}\right)$, de pouco significado prático e explicada pela baixa variabilidade de P no solo (Tabela 1).

Verificou-se, em outro trabalho, que, independentemente do método de amostragem para avaliação da fertilidade média do solo (média aritmética de amostras simples ou análise química da amostra composta) e do instrumento de 
Tabela 2. Teores de fósforo, potássio, cálcio e magnésio no solo, avaliados pela média aritmética de resultados de análises químicas de amostras simples e pela análise química da amostra composta, em função do número de amostras simples coletadas para formar uma amostra composta

\begin{tabular}{|c|c|c|}
\hline \multirow{2}{*}{$\begin{array}{l}\text { Número de } \\
\text { amostras simples } \\
\text { por amostra } \\
\text { composta }\end{array}$} & \multicolumn{2}{|c|}{ Forma de avaliação do teor médio do nutriente no solo } \\
\hline & Média aritmética ${ }^{(1)}$ & Amostra composta ${ }^{(2)}$ \\
\hline \multicolumn{3}{|c|}{ Teor de fósforo disponível (mg dm³) } \\
\hline 4 & 2,31 & 2,45 \\
\hline 8 & 2,25 & 2,22 \\
\hline 14 & 2,29 & 2,45 \\
\hline 20 & 2,29 & 2,45 \\
\hline 28 & 2,28 & 2,45 \\
\hline 36 & 2,27 & 2,45 \\
\hline Média(3) & $2,28 \mathrm{~b}$ & $2,42 a$ \\
\hline Modelo ajustado: & $\hat{y}=\bar{y}=2,28$ & $\hat{y}=\bar{y}=2,42$ \\
\hline \multicolumn{3}{|c|}{ Teor de potássio disponível (mg dm³) } \\
\hline 4 & 29,06 & 25,16 \\
\hline 8 & 25,64 & 26,25 \\
\hline 14 & 28,55 & 27,89 \\
\hline 20 & 26,87 & 25,70 \\
\hline 28 & 28,49 & 26,79 \\
\hline 36 & 29,42 & 27,89 \\
\hline Média(3) & $28,00 a$ & $26,61 a$ \\
\hline Modelo ajustado: & $\hat{y}=\bar{y}=28,00$ & $\hat{y}=\bar{y}=26,61$ \\
\hline \multicolumn{3}{|c|}{ Teor de cálcio trocável $\left(\mathrm{cmol}_{\mathrm{c}} \mathrm{dm}^{-3}\right)$} \\
\hline 4 & 0,63 & 0,58 \\
\hline 8 & 0,57 & 0,52 \\
\hline 14 & 0,72 & 0,65 \\
\hline 20 & 0,69 & 0,52 \\
\hline 28 & 0,69 & 0,58 \\
\hline 36 & 0,76 & 0,62 \\
\hline Média(3) & $0,67 a$ & $0,58 \mathrm{~b}$ \\
\hline Modelo ajustado: & $\hat{y}=\bar{y}=0,67$ & $\hat{y}=\bar{y}=0,58$ \\
\hline \multicolumn{3}{|c|}{ Teor de mágnésio trocável $\left(\mathrm{cmol}_{\mathrm{c}} \mathrm{dm}^{-3}\right)$} \\
\hline 4 & 0,52 & 0,82 \\
\hline 8 & 0,47 & 0,72 \\
\hline 14 & 0,47 & 0,68 \\
\hline 20 & 0,47 & 0,62 \\
\hline 28 & 0,46 & 0,62 \\
\hline 36 & 0,46 & 0,55 \\
\hline Média(3) & $0,48 \mathrm{~b}$ & $0,67 a$ \\
\hline Modelo ajustado: & (4) & (5) \\
\hline
\end{tabular}

${ }^{(1)}$ Analisou-se quimicamente cada amostra simples separadamente e se calculou a média aritmética dos resultados. Os resultados apresentados são médias de três repetições

(2)Analisou-se quimicamente apenas uma amostra composta. Os resultados apresentados são médias de três repetições

${ }^{(3)}$ Médias seguidas de mesma letra na linha não diferem entre si pelo teste $\mathrm{F}$. (4) $\hat{y}=0,4556+0,22477 * / \mathrm{NAS}$, com $\mathrm{R}^{2}=0,90$.

(5) $\hat{y}=0,5684+1,0533 * / \mathrm{NAS}$, com $\mathrm{R}^{2}=0,90$. NAS=número de amostras simples. *Significativo a $5 \%$ de probabilidade

coleta das amostras (pá de corte ou trado de caneca), o número de amostras simples para formar uma amostra composta não teve influência na estimativa dos teores médios de todas as características químicas avaliadas, tanto para o plantio direto como para o plantio convencional (Olivei-
Tabela 3. Teores de alumínio trocável e de matéria orgânica no solo e valor de pH do solo, avaliados pela média aritmética de resultados de análises químicas de amostras simples e pela análise química da amostra composta, em função do número de amostras simples coletadas para formar uma amostra composta

\begin{tabular}{|c|c|c|}
\hline \multirow{2}{*}{$\begin{array}{c}\text { Número de } \\
\text { amostras simples } \\
\text { por amostra } \\
\text { composta }\end{array}$} & \multicolumn{2}{|c|}{ Forma de avaliação do teor médio do nutriente no solo } \\
\hline & Média aritmética ${ }^{(1)}$ & Amostra composta ${ }^{(2)}$ \\
\hline \multicolumn{3}{|c|}{ Teor de alumínio trocável $\left(\mathrm{cmol}_{\mathrm{c}} \mathrm{dm}^{-3}\right)$} \\
\hline 4 & 27,35 & 32,87 \\
\hline 8 & 26,51 & 30,67 \\
\hline 14 & 26,48 & 32,52 \\
\hline 20 & 27,27 & 29,99 \\
\hline 28 & 26,59 & 30,33 \\
\hline 36 & 27,23 & 30,88 \\
\hline Média(3) $^{(3)}$ & $26,90 \mathrm{~b}$ & $31,21 a$ \\
\hline Modelo ajustado: & $\hat{y}=\bar{y}=26,90$ & $\hat{y}=\bar{y}=31,21$ \\
\hline \multicolumn{3}{|c|}{ Teor de matéria orgânica $\left(\mathrm{g} \mathrm{kg}^{-1}\right)$} \\
\hline 4 & 27,35 & 32,87 \\
\hline 8 & 26,51 & 30,67 \\
\hline 14 & 26,48 & 32,52 \\
\hline 20 & 27,27 & 29,99 \\
\hline 28 & 26,59 & 30,33 \\
\hline 36 & 27,23 & 30,88 \\
\hline Média $^{(3)}$ & $26,90 \mathrm{~b}$ & $31,21 a$ \\
\hline Modelo ajustado: & $\hat{y}=\bar{y}=26,90$ & $\hat{y}=\bar{y}=31,21$ \\
\hline \multicolumn{3}{|c|}{$\mathrm{pH}$} \\
\hline 4 & 4,81 & 4,90 \\
\hline 8 & 4,73 & 4,73 \\
\hline 14 & 4,79 & 4,63 \\
\hline 20 & 4,79 & 4,77 \\
\hline 28 & 4,77 & 4,80 \\
\hline 36 & 4,80 & 4,80 \\
\hline Média(3) & $4,78 a$ & 4,77 a \\
\hline Modelo ajustado: & $\hat{y}=\bar{y}=4,78$ & (6) \\
\hline
\end{tabular}

${ }^{(1)}$ Analisou-se quimicamente cada amostra simples separadamente e se calculou a média aritmética dos resultados. Os resultados apresentados são médias de três repetições

${ }^{(2)}$ Analisou-se quimicamente apenas uma amostra composta. Os resultados apresentados são médias de três repetições

${ }^{(3)}$ Médias seguidas de mesma letra na linha não diferem entre si pelo teste $\mathrm{F}$ (6) $\hat{y}=4,9091-0,02 * \mathrm{NAS}+0,0005^{*}(\mathrm{NAS})^{2}$, com $\mathrm{R}^{2}=0,40$. NAS=número de amostras simples. *Significativo a $5 \%$ de probabilidade

ra et al., 2007). Com exceção do $\mathrm{Mg}^{2+}$, para o qual a diferença entre os métodos foi de $40 \%$, para as demais características químicas avaliadas, em que os métodos foram diferentes, esta diferença foi no máximo $16 \%$ (Tabelas 2 e 3); ante esta pequena magnitude de diferença entre os métodos e se considerando a ampla faixa de valores dessas características consideradas na interpretação das análises de solos (Ribeiro et al., 1999), na prática os dois métodos levariam, na maioria das vezes, à mesma recomendação de calagem e adubação para as culturas; neste sentido, pode-se afirmar que os dois métodos são semelhantes.

Com exceção do $\mathrm{Mg}^{2+}$ avaliado pelos dois métodos (Tabela 2) e do $\mathrm{pH}$ avaliado a partir da análise química da amos- 
tra composta (Tabela 3), as médias dos valores das características químicas determinadas não foram influenciadas pelo aumento do número de amostras simples para formar uma amostra composta representativa, indicando que, se se coletarem quatro ou trinta e seis amostras simples, as médias dessas características não serão diferentes, independente do método de avaliação da fertilidade média do solo (Tabelas 2 e 3). Nos casos do $\mathrm{Mg}^{2+}$ e do $\mathrm{pH}$, a coleta de apenas quatro amostras simples resultou em valores mais elevados dessas características químicas mas, quando este número variou de oito a trinta e seis amostras simples, praticamente não se observou variação das médias dos valores dessas características químicas (Tabelas 2 e $3)$; este comportamento é semelhante ao verificado por Oliveira et al. (2007), os quais afirmam que a coleta de maior número de amostras simples não exerce influência no valor médio da característica química do solo avaliada mas aumenta a confiabilidade ou exatidão dessa média obtida, pela diminuição do erro obtido em torno da média verdadeira; portanto, a coleta de pelo menos oito amostras simples de solo seria suficiente para formar uma amostra composta representativa para avaliação da fertilidade média do solo de uma unidade de amostragem aparentemente homogênea, porém, a confiabilidade ou exatidão da estimativa dessa fertilidade média será maior quanto maior for o número de amostras simples coletadas para formar uma amostra composta.

\section{CONCLUSÕES}

1. A variabilidade das características químicas do solo praticamente não variou com a diminuição do tamanho da área amostrada.

2. Na prática da interpretação de análise de solo para recomendação de calagem e adubação, as doses de calcário e fertilizante devem ser as mesmas, independentemente se a fertilidade média do solo foi avaliada a partir da análise química da amostra composta ou a partir da média aritmética dos resultados das análises químicas.

3. A coleta de pelo menos oito amostras simples de solo seria suficiente para formar uma amostra composta representativa para avaliação da fertilidade média do solo de uma unidade de amostragem aparentemente homogênea, mas a confiabilidade ou exatidão da estimativa dessa fertilidade média será maior quanto maior for o número de amostras simples coletadas para formar uma amostra composta.

\section{LITERATURA CITADA}

Alvarez V., V. H.; Carraro, I. M. Variabilidade do solo numa unidade de amostragem em solos de Cascavel e de Ponta Grossa, Paraná. Revista Ceres, v.23, p.503-510, 1976.

Alvarez V., V. H.; Guarçoni M., A. Variabilidade horizontal da fertilidade do solo de uma unidade de amostragem em sistema plantio direto. Revista Brasileira de Ciência do Solo, v.27, p.297-310, 2003.
Barreto, A. C.; Novais, R. F.; Braga, J. M. Determinação estatística do número de amostras simples de solo por área para avaliação de sua fertilidade. Revista Ceres, v.21, p.142-147, 1974.

Cantarutti, R. B.; Barros, N. F.; Martinez, H. E. P.; Novais, R. F. Avaliação da fertilidade de solo e recomendação de fertilizantes. In: Novais, R. F.; Alvarez V., V. H.; Barros, N. F.; Fontes, R. L. F.; Cantarutti, R. B.; Neves, J. C. L. (ed.). Fertilidade do solo. Viçosa: SBCS, 2007. p.769-850.

Guarçoni M., A.; Alvarez V., V. H.; Novais, R. F.; Cantarutti, R. B.; Leite, H. G.; Freire, F. M. Definição da dimensão do indivíduo solo e determinação do número de amostras simples necessário à sua representação. Revista Brasileira de Ciência do Solo, v.30, p.943-954, 2006.

Guarçoni M., A.; Alvarez V., V. H.; Novais, R. F.; Cantarutti, R. B.; Leite, H. G.; Freire, F. M. Diâmetro de trado necessário à coleta de amostras num Cambissolo sob plantio direto ou sob plantio convencional antes ou depois da aração. Revista Brasileira de Ciência do Solo, v.31, p.947-959, 2007.

James, D. W.; Wells, K. L. Soil sample collection and handling: Technique based on source and degree of field variability. 3.ed. In: Westerman, R. L. (ed.). Soil testing and plant analysis. Madison: Soil Science Society of America, 1990. p.25-44.

Machado, L. O.; Lana, A. M. Q; Lana, R. M. Q; Guimarães, E. C.; Ferreira, C. V. Variabilidade espacial de atributos químicos do solo em áreas sob sistema plantio convencional. Revista Brasileira de Ciência do Solo, v.31, p.591-599, 2007.

Mello, G.; Bueno, C. R. P.; Pereira, G. T. Variabilidade espacial das propriedades físicas e químicas do solo em áreas intensamente cultivadas. Revista Brasileira de Engenharia Agrícola e Ambiental, v.10, n.2, p.294-305, 2006.

Oliveira, F. H. T.; Arruda, J. A.; Silva, I. F.; Alves, J. C. Amostragem para avaliação da fertilidade do solo em função do instrumento de coleta das amostras e de tipos de preparo do solo. Revista Brasileira de Ciência do Solo, v.31, p.973-983, 2007.

Oliveira, F. H. T.; Novais, R. F.; Alvarez V., V. H.; Cantarutti, R. B.; Barros, N. F. Fertilidade do solo no sistema plantio direto. In: Alvarez V., V. H.; Schaefer, C. E. G. R; Barros, N. F.; Mello, J. W. V.; Costa, L. M. (ed.). Tópicos em ciência do solo. v.2, Viçosa: SBCS, 2002. p.393-486.

Raij, B. van. Fertilidade do solo e adubação. Piracicaba: Ceres, 1991. 343p.

Raun, W. R.; Solie, J. B.; Johnson, G. V.; Stone, M. L.; Whitney, R. W.; Lees, H. L.; Sembiring, H.; Phillips, S. B. Microvariability in soil test, plant nutrient, and yield parameters in bermudagrass. Soil Science Society American Journal, v.62, p.683-690, 1998.

Ribeiro, A. C.; Guimarães, P. T. G.; Alvarez V., V. H. Recomendações para o uso de corretivos e fertilizantes em Minas Gerais: $5^{\mathrm{a}}$ aproximação. Viçosa: CFSEMG, 1999. 359p.

Saraiva, O. F.; Alvarez V., V. H.; Costa, L. M. Variabilidade de algumas características físicas e químicas de um Podzólico VermelhoAmarelo Câmbico distrófico. Revista Ceres, v.39, p.529-541, 1992.

Schlindwein, J. A.; Anghinoni, I. Variabilidade horizontal de atributos de fertilidade e amostragem do solo no sistema plantio direto. Revista Brasileira de Ciência do Solo, v.24, p.85-91, 2000.

Silva, F. M. S.; Souza, Z. M.; Figueirêdo, C. A. P.; Marques Júnior, J.; Machado, R. V. Variabilidade espacial de atributos químicos e de produtividade na cultura do café. Ciência $\mathrm{Ru}-$ ral, v.37, n.2, p.401-407, 2007. 